\title{
September 2015 Imaging Case of the Month
}

\author{
Philip W. Ho, M.D. \\ Clinton Jokerst, M.D. \\ Department of Medical Imaging \\ Banner University Medical Center \\ Tucson, AZ
}

Clinical History: A 51-year-old white man with a past medical history significant for weight, loss, hypertension and a 60 pack-year smoking history presented to the emergency department with hemoptysis and chest pain. He was afebrile with an unremarkable CBC. Frontal chest radiography (Figure 1) was obtained.

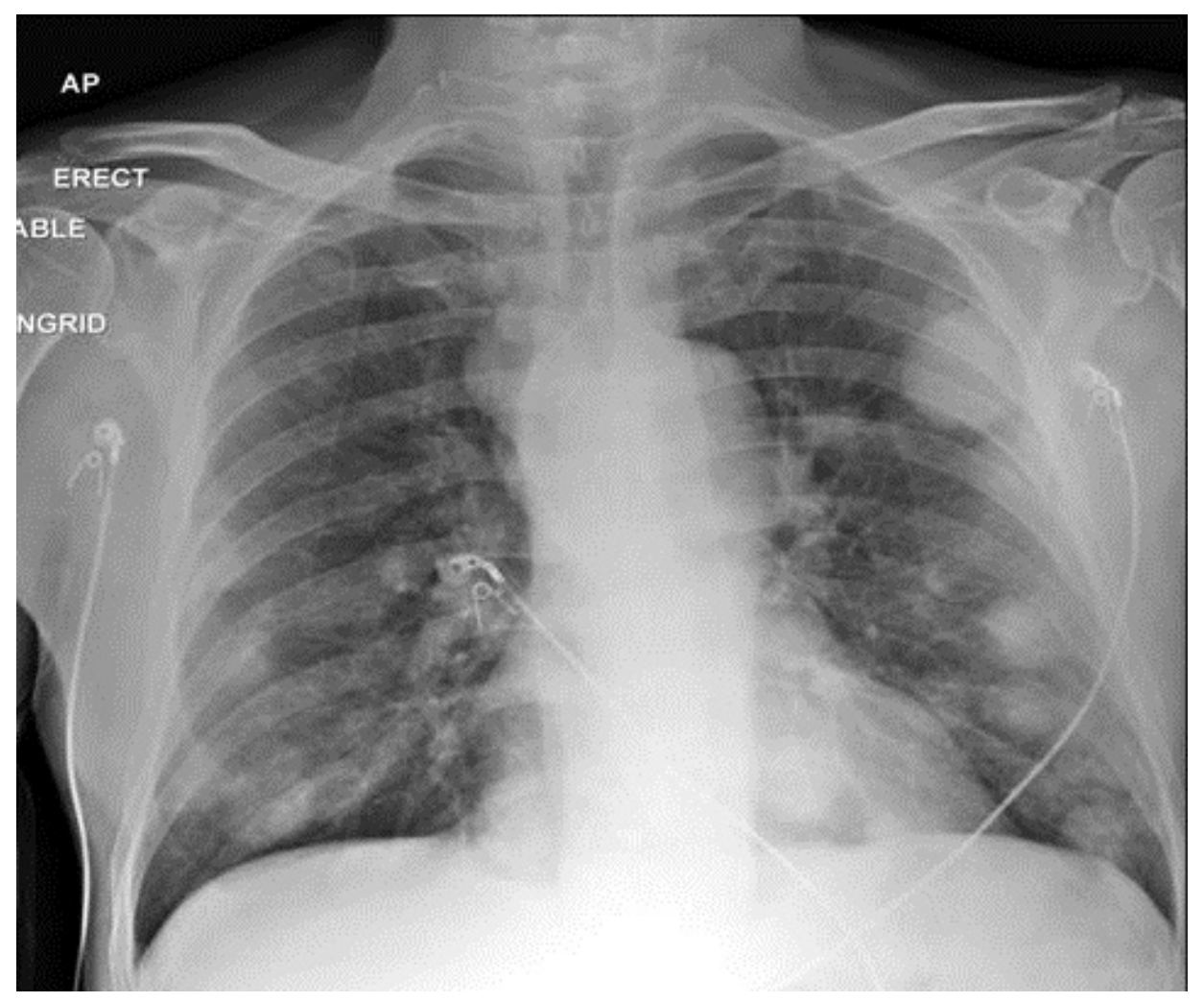

Figure 1. Frontal chest radiography.

There are multiple large pulmonary nodules scattered throughout both lungs. Which is the least likely diagnosis?

1. Fungal infection

2. Granulomatosis with polyangiitis (GPA)

3. Metastatic disease

4. Septic emboli 


\section{Correct! \\ 2. Granulomatosis with polyangiitis}

All the choices could present with multiple pulmonary nodules, but there is also an enlarged mediastinum, concerning for a mediastinal mass/lymphadenopathy, which GPA typically doesn't present with. The other three choices could have involvement of the mediastinum and differentiating between them solely on chest radiography is difficult, although septic emboli may be expected to show cavitation within some of the nodules, which is not present.

Contrast-enhanced thoracic CT (Figure 2) was performed for further evaluation of the findings seen at chest radiography.

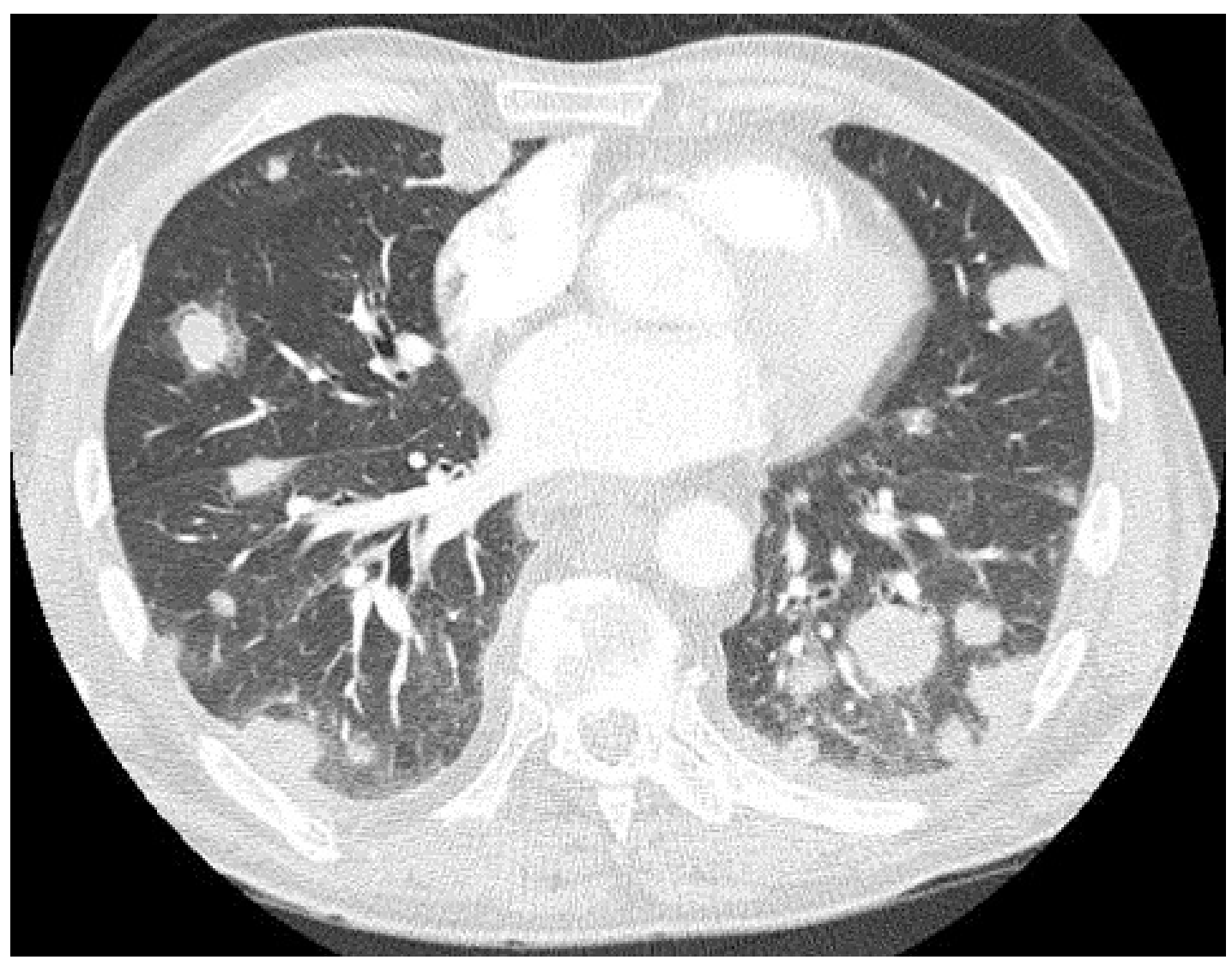

Figure 2. Enhanced thoracic CT scan in lung windows.

The pulmonary nodules are identified as being solid with surrounding ground-glass opacity (CT halo sign). What classically does the halo represent?
1. Edema
2. Hemorrhage
3. Infarction.
4. Tumor 


\section{Correct! \\ 2. Hemorrhage}

The ground-glass opacity surrounding the pulmonary nodules classically refers to hemorrhagic nodules and was first described with invasive aspergillosis in severely immunocompromised patients. Hemorrhagic nodules can also be found with metastatic disease from hypervascular malignancies such as angiosarcoma, choriocarcinoma, thyroid, renal cell carcinoma, melanoma, and Kaposi's sarcoma. Granulomatosis with polyangiitis can also present with hemorrhagic pulmonary nodules. In addition to hemorrhage, tumor infiltration with lepidic growth patterns such as with adenocarcinoma can demonstrate the CT halo sign, but was not classically described.

Enhanced thoracic CT displayed in soft tissue windows also showed that some of the pulmonary nodules were hyperattenuating, possibly reflecting calcification, such as the left lower lobe pulmonary nodule shown in Figure 3.

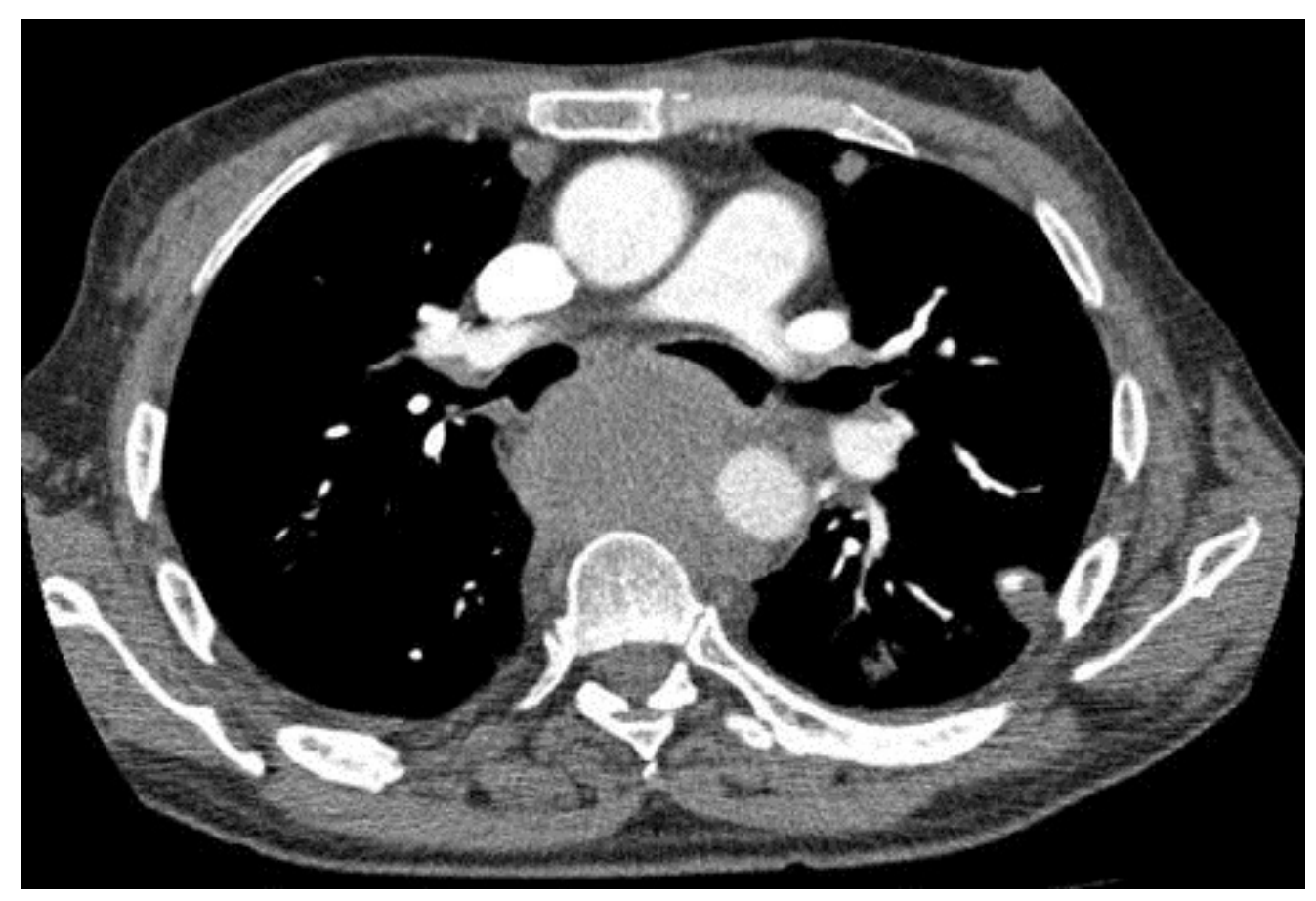

Figure 3. Enhanced thoracic CT displayed in soft tissue windows.

Which of the following is not within the differential diagnostic considerations for calcified metastatic pulmonary nodules?

1. Chondrosarcomas

2. Melanoma

3. Mucin-producing carcinomas

4. Osteosarcomas 


\section{Correct!}

\section{Melanoma}

Calcified pulmonary nodules have a wide differential. There are benign causes such as granulomas, occupational exposure such as silicosis, and treated infections such as varicella. There are also malignant causes, including any osteoid matrix- or chondroid matrix- producing tumor, such as osteosarcoma and chrondrosarcoma, respectively. Also, mucin producing tumors of the gastrointestinal tract or breast can lead to mucoid calcification. Treated metastatic disease can also calcify in the case of choriocarcinoma and thyroid malignancies. Melanoma is a hypervascular tumor, but typically does not calcify. Note there is also a large middle mediastinal mass, which represents massive lymphadenopathy.

Review of the entire thoracic CT showed that only one nodule contained a focus of calcification; the remainder of the numerous, bilateral nodules were non-calcified. The patient subsequently developed abdominal pain and contrast-enhanced CT (CECT) of the abdomen (Figure 4) was performed for further evaluation.

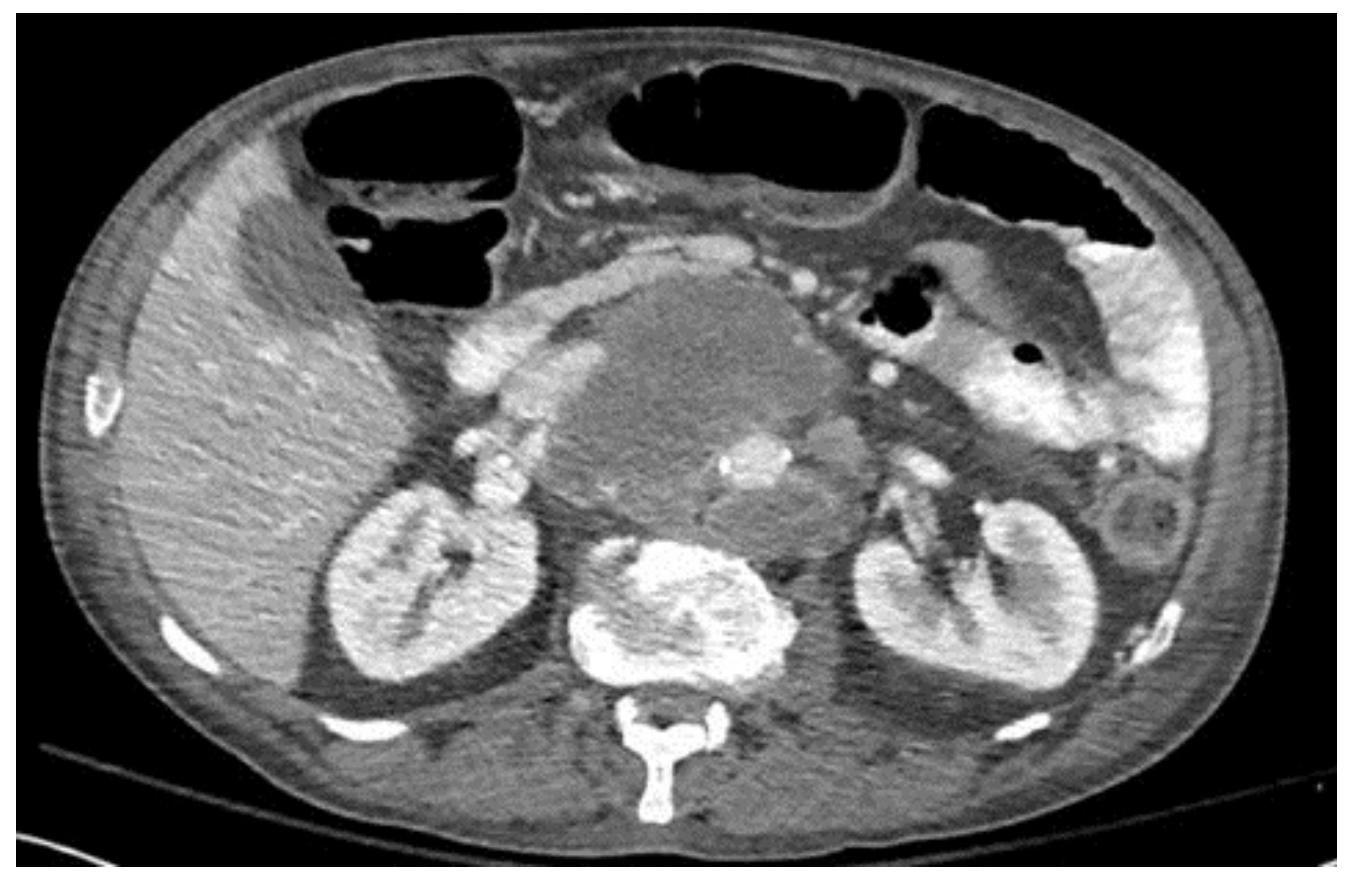

Figure 4. Enhanced abdominal CT displayed in soft tissue windows.

There is massive retroperitoneal lymphadenopathy. Taking into account patient's clinical presentation and the findings in the chest, what is the most likely diagnosis?

1. Lymphoma

2. Metastatic choriocarcinoma

3. Metastatic renal cell carcinoma

4. Tuberculosis 


\section{Correct! \\ 2. Metastatic choriocarcinoma}

The patient's overall presentation and imaging findings are concerning for metastatic disease, as he is afebrile with weight loss with multiple pulmonary nodules and lymphadenopathy in both the chest and abdomen. Tuberculosis is unlikely as he is afebrile and the imaging findings are atypical for this diagnosis. Lymphoma is certainly a consideration given the lymphadenopathy, but the multiple large pulmonary nodules would be unusual for this diagnosis. Given the CT halo sign, a hypervascular malignancy is favored; however, the patient's kidneys appeared unremarkable. In a male patient, a metastatic germ cell malignancy could present with variably-sized pulmonary nodules and masses as well as lymph node enlargement, particularly retroperitoneal lymphadenopathy.

The patient's beta-hCG was found to be markedly elevated at 130,000 and there was concern for a metastatic germ cell tumor. Eventually, one of the lung nodules was biopsied under CT guidance and pathology was consistent with metastatic choriocarcinoma.

Diagnosis: Metastatic choriocarcinoma with lymphadenopathy and hemorrhagic pulmonary metastases

\section{References}

1. Boitsios G, Bankier AA, Eisenberg RL. Diffuse pulmonary nodules. AJR Am J Roentgenol. 2010;194:W354-66. [CrossRef] [PubMed]

2. Marchiori E, Souza AS Jr, Franquet T, Müller NL. Diffuse high-attenuation pulmonary abnormalities: a pattern-oriented diagnostic approach on high-resolution CT. AJR Am J Roentgenol. 2005;184:273-82. [CrossRef] [PubMed]

3. Pinto PS. The CT halo sign. Radiology. 2004;230(1):109-10. [CrossRef] [PubMed] 